\title{
Saying it with feeling: neural responses to emotional vocalizations
}

\author{
J.S. Morris ${ }^{\mathrm{a}}$, S.K. Scott ${ }^{\mathrm{b}}$, R.J. Dolan ${ }^{\mathrm{a}, \mathrm{c}, *}$ \\ ${ }^{a}$ Wellcome Department of Cognitive Neurology, Queen Square, London WC1N 3BG, UK \\ ${ }^{\mathrm{b}}$ Institute of Cognitive Neuroscience, Alexandra House, 17 Queen Square, London WC1N $3 A R, U K$ \\ ${ }^{\mathrm{c}}$ Royal Free and University College Hospitals School of Medicine, Rowland Hill Street, London NW3 2DF, UK \\ Received 2 July 1998; accepted 9 December 1998
}

\begin{abstract}
To determine how vocally expressed emotion is processed in the brain, we measured neural activity in healthy volunteers listening to fearful, sad, happy and neutral non-verbal vocalizations. Enhanced responses to emotional vocalizations were seen in the caudate nucleus, as well as anterior insular, temporal and prefrontal cortices. The right amygdala exhibited decreased responses to fearful vocalizations as well as fear-specific inhibitory interactions with left anterior insula. A region of the pons, implicated in acoustic startle responses also showed fear-specific interactions with the amygdala. The data demonstrate: firstly, that processing of vocal emotion involves a bilaterally distributed network of brain regions; and secondly, that processing of fear-related auditory stimuli involves context-specific interactions between the amygdala and other cortical and brainstem regions implicated in fear processing. (C) 1999 Elsevier Science Ltd. All rights reserved.
\end{abstract}

Keywords: Emotion; Prosody; Amygdala; Insula; Fear; Acoustic startle

\section{Introduction}

Studies of patients with focal brain damage implicate temporal cortex, insula, basal ganglia and ventral prefrontal cortex in the perception and expression of vocal emotion [6,7,13,15-17,23,34,35]. Right hemisphere specialization for vocal emotion processing has been proposed on the basis of right posterior (Wernicke-like) lesions being associated with sensory aprosodias, and right anterior (Broca-like) damage producing expressive deficits [13,35]. However, problems with vocal emotion have also been reported with brain pathologies not conforming to this right hemispheric, anterior-posterior categorization $[6,7,5,17$, 27, 34]. Moreover, a functional imaging study of receptive emotional prosody found activations in right ventral prefrontal cortex rather than the posterior areas predicted by Gorelick and Ross's (1988) model [13]. These data suggest that the neural processing of vocal

\footnotetext{
* Corresponding author. Fax: +44-171-813-4120.

E-mail address: r.dolan@fil.ion.fil.ucl.ac.uk (R.J. Dolan)
}

emotion may have a more complex anatomical distribution than originally proposed. This is congruent with the complexity of paralinguistic and prosodic emotional cues expressed in human vocalizations.

Bilateral amygdala damage has been associated with selectively impaired recognition of fearful and angry vocalizations [37], providing evidence for functional specialization within neural systems processing vocal emotion. The amygdala, which is implicated in fear conditioning $[5,20,21,24]$, modulation of startle responses to fear-conditioned stimuli [8] and perception of fearful facial expressions $[1,4,25,28]$, appears to play a crucial multimodal role in fear processing. There are extensive anatomical connections between the amygdala and prosody-related brain regions, e.g. insula, basal ganglia, temporal and ventral prefrontal cortices [2], consistent with the suggestion that an interacting network of brain regions is engaged in processing vocal emotion. We are not aware of any study, however, that has addressed the issue of the amygdala's functional interactions during auditory affective processing.

In this study we used positron emission tomography 
Table 1

Brain regions with (A) enhanced and (B) decreased responses to emotional (fearful, happy and sad) voices compared to neutral. Coordinates of maximally activated voxels, $Z$ scores and $P$ values are shown

\begin{tabular}{lcrr}
\hline & Coordinates & $Z$ score & $P$ value \\
\hline (A) Increased responses & & & $<0.001$ \\
L. mid. temporal gyrus & $-62,-6,-4$ & 3.37 & 0.001 \\
L. sup. frontal gyrus & $0,54,42$ & 3.18 & 0.001 \\
L. ventral prefrontal cortex & $-36,30,-14$ & 2.98 & 0.002 \\
L. ant. insula & $-34,12,-16$ & 2.92 & 0.003 \\
R. ventral prefrontal cortex & $34,36,-12$ & 2.71 & 0.004 \\
R. ant. insula & $46,20,-2$ & 2.65 & 0.013 \\
& $42,12,4$ & 2.22 & 0.004 \\
R. caudate nucleus & $14,6,10$ & 2.62 & $<0.001$ \\
& & & $<0.001$ \\
(B) Decreased responses & & 3.88 & $<.001$ \\
L. insula & $-32,-12,26$ & 3.88 & $<0.001$ \\
R. insula & $36,-4,22$ & 3.65 & $<.001$ \\
L. mid. temporal gyrus & $-60,-56,-8$ & 3.46 & \\
L. inf. temporal gyrus & $-52,-18,-26$ & 3.34 \\
R. fusiform gyrus & $32,-44,-18$ & & $<$ \\
\end{tabular}

(PET) to measure neural activity during presentation of sad, happy, fearful and neutral non-verbal vocalizations. Male and female vocalizations were included in each emotional category and subjects were required to perform a sex decision task (i.e. indicate male or female) for each individual vocal presentation. Subjects were not explicitly informed of the emotional experimental variable. On the basis of previous lesion studies $[6,7,13,15-17,23,34,35]$ differential activation in regions such as insula, basal ganglia, temporal and ventral prefrontal cortices was expected during the emotional conditions. Also, a specific response in the amygdala in relation to fearful voices was expected in view of its role in fear processing.

\section{Methods}

Six right-handed male subjects (mean age 32.7 years) took part in the study. All subjects were unmedicated, and had no past history of neurological or psychiatric illness. Informed consent was obtained and the study was approved by the local hospital ethics committee and ARSAC(UK). Each subject had 12 scans of the distribution of $\mathrm{H}_{2}^{15} \mathrm{O}$ which were obtained using a Siemens/CPS ECAT EXACT HR + PET Scanner operated in high sensitivity 3-D mode. Subjects received a total of $350 \mathrm{MBq}$ of $\mathrm{H}_{2}^{15} \mathrm{O}$ over $20 \mathrm{~s}$ through a forearm cannula. Images were reconstructed into 63 planes, using a Hanning filter, resulting in a $6.4 \mathrm{~mm}$ transaxial and $5.7 \mathrm{~mm}$ axial resolution (full width half maximum). Each scanning window was of $90 \mathrm{~s}$ duration.

Non verbal, neutral sounds (voiced nasals) were produced by two native English speakers (one male and one female) at different pitches and digitized. These matched the overall duration (range 1-2 s) and frequency content of happy, sad and fearful vocalizations from the same speakers which have been described in a previous report [37]. During scanning, 10 vocal stimuli from one of the four emotional categories were presented every 6 seconds while subjects performed a sex decision task ('male' or 'female') by pressing one of two buttons with their right hand. Post-scanning, 20 of the emotional sounds (5 from each category) were presented again and subjects categorized them as either 'happy', 'sad', 'fearful', 'neutral' or 'other' (specified by the subject).

The PET data were analyzed using statistical parametric mapping (SPM96) software from the Wellcome Department of Cognitive Neurology, London. After initial realignment, the PET scans were transformed into a standard stereotactic space. Structural MRIs from each subject were co-registered into the same space. The scans were then smoothed using a Gaussian filter set at $12 \mathrm{~mm}$ full width at half maximum. The $r \mathrm{CBF}$ measurements were adjusted to a global mean of $50 \mathrm{ml} / \mathrm{dl} / \mathrm{min}$. A blocked (by subject) Ancova model was fitted to the data at each voxel, with condition effects for each of the 4 emotional conditions, and global $\mathrm{CBF}$ as a confounding covariate. Predetermined contrasts of the condition effects at each voxel were assessed using a $t$-statistic, giving a statistic image for each contrast.

In subsequent regression analyses, the adjusted $r \mathrm{CBF}$ values at the maximal foci of activation in cortical and subcortical areas of interest were grouped into fearful and non-fearful (happy, sad, and neutral) conditions. These two sets of values were then used as covariates of interest in a separate analysis testing for 
a
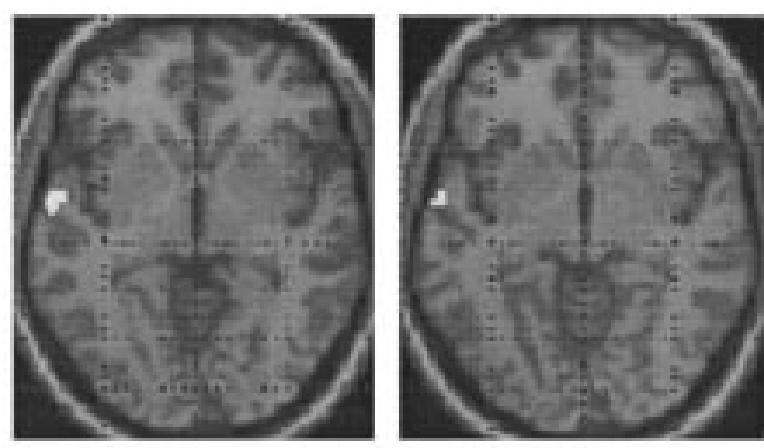

C
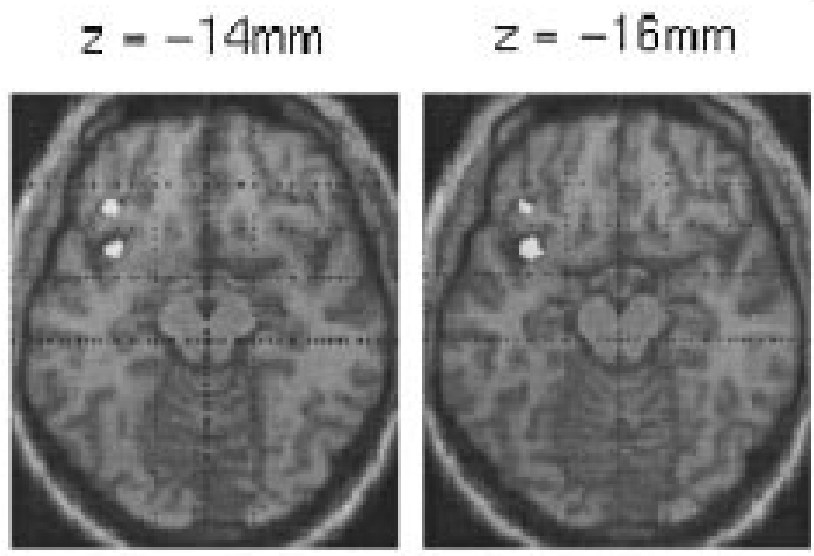

b

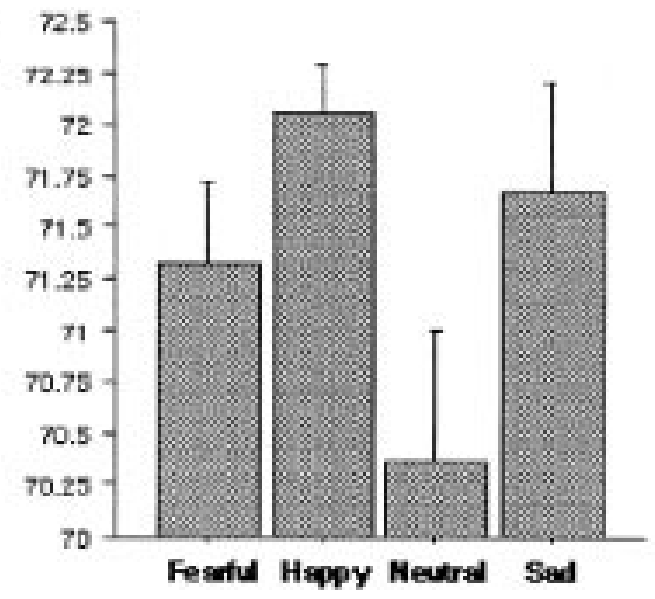

d

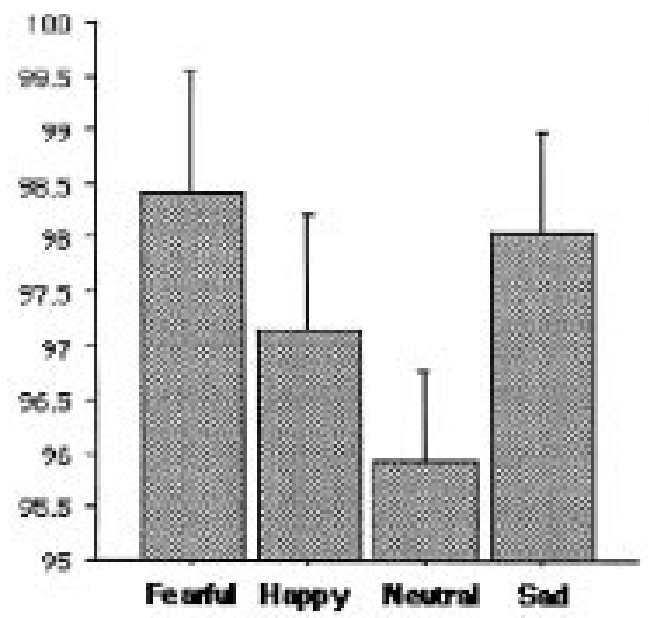

Fig. 1. (a) An SPM showing a region in left middle temporal gyrus with enhanced responses in the contrast of emotional versus non-emotional conditions. The activation is displayed on transverse slices $(z=4$ and $-6 \mathrm{~mm})$ of a canonical MRI. An uncorrected $P$ value of 0.01 was used as the threshold for the contrast; (b) mean $r \mathrm{CBF}$ values in each of the 4 conditions for the maximal voxel $(x=62, y=-6, z=-4)$ of the region displayed in (a). Bars represent 2 standard errors; (c) an SPM showing regions in left anterior insula and ventral prefrontal cortex with enhanced responses in the contrast of emotional vs non-emotional conditions. The activation is displayed on transverse slices $(z=14$ and $-16 \mathrm{~mm})$ of a canonical MRI; (d) mean $r$ CBF values in each of the 4 conditions for the maximal voxel $(x=-34, y=12, z=-16)$ in the anterior insula displayed in (c). Bars represent 2 standard errors.

pyschophysiological interactions [10]. The condition blocks and the global CBF values were used as confounding covariates. Differences between the regression slopes obtained for the covariates of interest (i.e. fearful vs non-fearful) were tested directly at every voxel to produce an $\operatorname{SPM}(\mathrm{t})$ showing brain areas in which the regression of activity with the region of interest changed significantly as a function of emotional category. The general methods employed by SPM are described in detail by Friston et al. [11].

\section{Results}

In the explicit sex decision task, all subjects performed close to ceiling ( $>90 \%$ correct). In emotion identification tests conducted post-scanning the overall identification rate was $80.12 \%$, with individual scores of $100 \%$ for happy, $70 \%$ for fearful, $75 \%$ for sad, and $80 \%$ for neutral. In post-scan debriefing, no subjects demonstrated any knowledge that the emotional content of the voices was critical to the experiment.

Compared to the neutral condition, emotional vocalizations (fearful, happy and sad) produced greater neural responses in left middle temporal gyrus (BA21), left superior frontal gyrus (BA9), right caudate nucleus, bilateral anterior insula, and bilateral ventral prefrontal cortex (BA11) (Table 1 and Fig. 1). Within these emotion-related regions (Table 1), a selectively increased response to fearful voices was seen in a region of the left anterior insula (Fig. 2a). Other brain regions with fear-related increases in activity are 


$$
z=-14 \mathrm{~mm}
$$

a

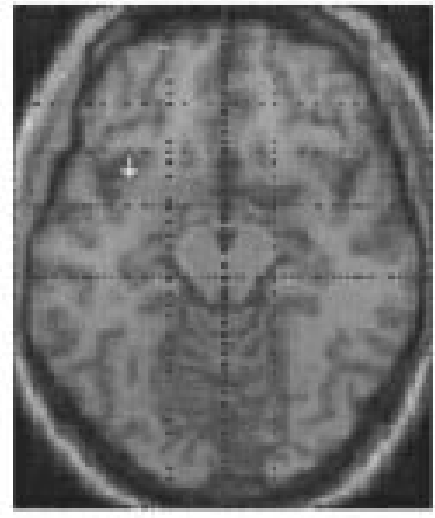

c

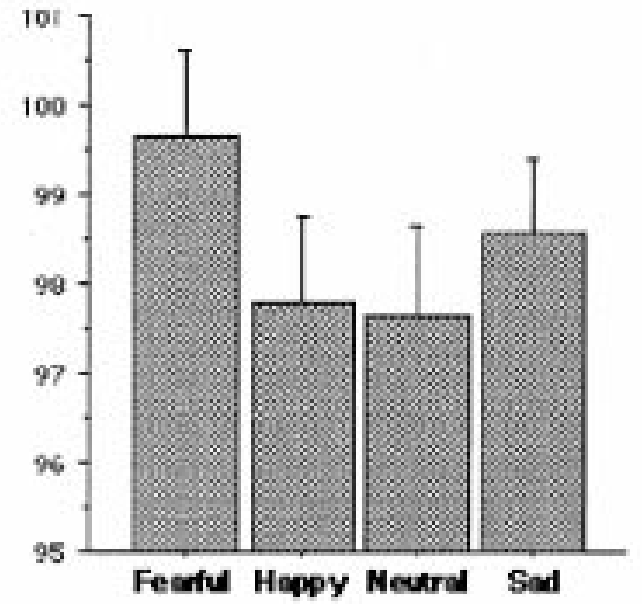

e

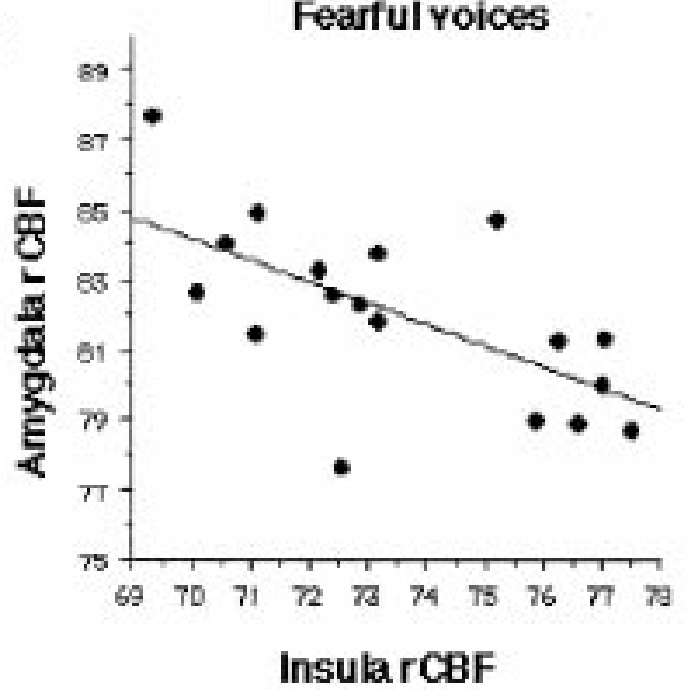

b

coronal

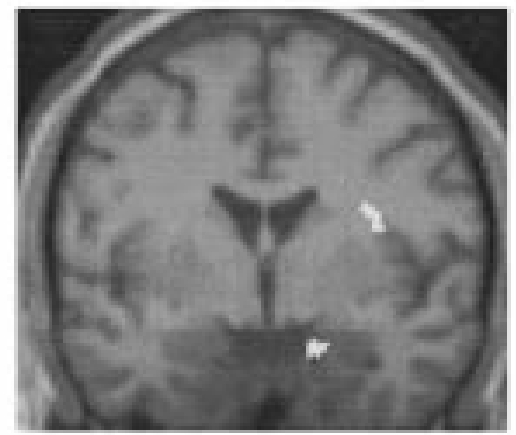

d

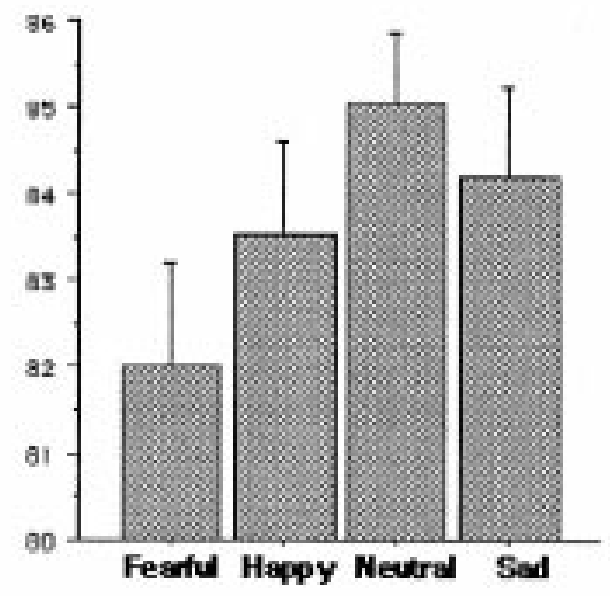

\section{Norfearful Yoices}

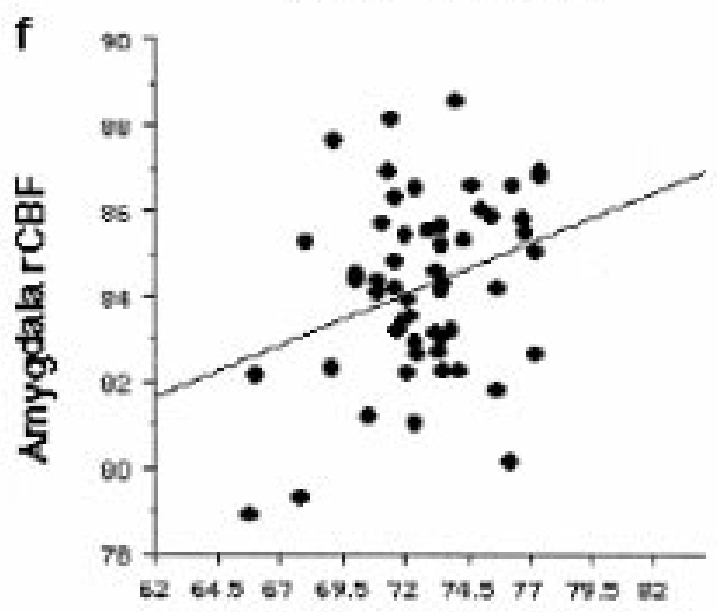

Insula rCBF

Fig. 2. (a) An SPM showing a region in left anterior insula with enhanced responses in the contrast of fearful vs all other conditions. The activation is displayed on a transverse slice $(z=-14 \mathrm{~mm})$ of a canonical MRI. An uncorrected $P$ value of 0.01 was used as the threshold for the contrast; (b) an SPM showing regions in right amygdala and right anterior insula with decreased responses in the contrast of fearful vs all other conditions. The activation is displayed on a coronal slice $(y=-4 \mathrm{~mm})$ of a canonical MRI; (c) mean $r \mathrm{CBF}$ values in each of the 4 conditions for the maximal voxel $(x=-34, y=18, z=-14)$ of the left anterior insula displayed in (a). Bars represent 2 standard errors; (d) mean $r$ CBF values in each of the 4 conditions for the maximal voxel $(x=18, y=-4, z=-22)$ in the right amygdala displayed in (b). Bars represent 2 standard errors; (e) bivariate regression plot of $r \mathrm{CBF}$ in the maximally activated voxels in left anterior insula (see (a) and (c)) and right amygdala (see (b) and (d)) during presentation of fearful voices; (f) bivariate regression plot of $r \mathrm{CBF}$ in the maximally activated voxels in left anterior insula (see (a) and (c)) and right amygdala (see (b) and (d)) during presentation of happy, sad and neutral voices. 
Table 2

Brain regions with (A) enhanced and (B) decreased responses to fearful voices compared to all other conditions (happy, sad and neutral). Coordinates of maximally activated voxels, $z$ scores and $P$ values are shown

\begin{tabular}{|c|c|c|c|}
\hline & Coordinates & $Z$ score & $P$ value \\
\hline \multicolumn{4}{|l|}{ (A) Increased responses } \\
\hline R. sup. frontal gyrus & $16,46,36$ & 3.17 & 0.001 \\
\hline R. ventral prefrontal cortex & $28,40,-24$ & 2.80 & 0.003 \\
\hline L. mid. temporal gyrus & $-72,-26,-10$ & 2.63 & 0.004 \\
\hline L. ant. insula & $-34,18,-14$ & 2.51 & 0.006 \\
\hline \multicolumn{4}{|l|}{ (B) Decreased responses } \\
\hline R. ant. insula & $34,10,20$ & 3.80 & $<0.001$ \\
\hline R. amygdala & $18,-4,-22$ & 3.09 & 0.001 \\
\hline
\end{tabular}

detailed in Table 2A. Activity in the right insula and right amygdala, in contrast, was selectively decreased in the fearful condition (Fig. 2b and Table 2B).

In order to determine whether processing of fear-related vocalizations is associated with fear-specific interactions within a network of brain regions, neural responses in the sole region where emotion-related activity was enhanced in the fearful condition (left anterior insula, maximal voxel $x=-34, y=18, z=-14$ ), were used as a predictor variable in a separate regression analysis [10]. In essence, this analysis identifies the presence of context-specific (i.e. fear-related) interactions between the insula and other brain regions. As demonstrated in Fig. 2e and $f$, a significant $(P<0.001)$ fear-specific interaction is present between left insula and right amygdala. The negative regression between these regions during fearful vocalizations (Fig. 2e) contrasts to the positive regression of activity in the other emotional conditions (Fig. 2f).

A similar regression analysis, using neural responses from the right amygdala (maximal voxel $x=18$, $y=-4, z=-22)$ as the covariate of interest, revealed a significant $(P<0.01)$ fear-specific interaction with a region of the pons encompassing the nucleus reticularis pontis caudalis (NRPC) (Fig. 3). A negative regression between amygdala and NRPC activity is present during fearful vocalizations (Fig. 3b) in contrast to the positive regression between these regions in all other conditions (Fig. 3c). The analysis demonstrates, therefore, that a change in covariation of amygdala and NRPC activity occurs specifically during the context of fearful vocalizations.

\section{Discussion}

The data from this study provide evidence for a more complex model of vocal emotional processing than that proposed from earlier lesion studies [13]. In particular, the results do not support the view that a non-dominant homologue of Wernicke's area is the only region specialized for perceiving emotion in the voice. Instead, we found that emotional vocalizations engaged structures such as the caudate nucleus, anterior insula, temporal and ventral prefrontal cortices across both hemispheres (Table 1). Although responses in these regions were relatively small, they generally accord with predictions based upon lesion data $[6,7,13,15-17,23,34-36]$. It is noteworthy that in a large study involving 46 patients with aprosodia, the brain regions most strongly implicated were in basal ganglia, anterior temporal lobe, insula and perisylvian cortex [6].

Profound non-verbal auditory agnosia and aprosodia, with preservation of other linguistic abilities, have been reported following circumscribed bilateral insula infarcts [15]. The left insula in particular appeared to be crucial in determining the receptive dysprosody seen in Habib et al.'s [15] study, and has also been implicated in expressive dysprosody and general speech apraxia [9]. The association with aprosodia is consistent with the insula's extensive reciprocal anatomical connections with auditory structures, including adjacent auditory cortex and medial geniculate nucleus, and its connections to regions such as amygdala, anterior cingulate, orbitofrontal, opercular and inferior frontal cortices that are implicated in affective and motivational functions [3]. Furthermore, animal studies suggest that the insula is involved in the temporal structuring of auditory perception in different behavioural contexts [14], a view consistent with a previous functional neuroimaging study in which the left insula (with left inferior Broca) was the only area activated when subjects discriminated sequences on the basis of rhythm [29]. A similar left insula response was seen in the present study even though subjects did not explicitly attend to the different rhythms in the stimuli. Since emotional sounds all contain temporally ordered substructures relevant to the identity of the sound, such rhythmic processing may be an obligatory aspect of the perception of these stimuli [26].

Whereas previous lesion $[13,16,36]$ and neuroima- 

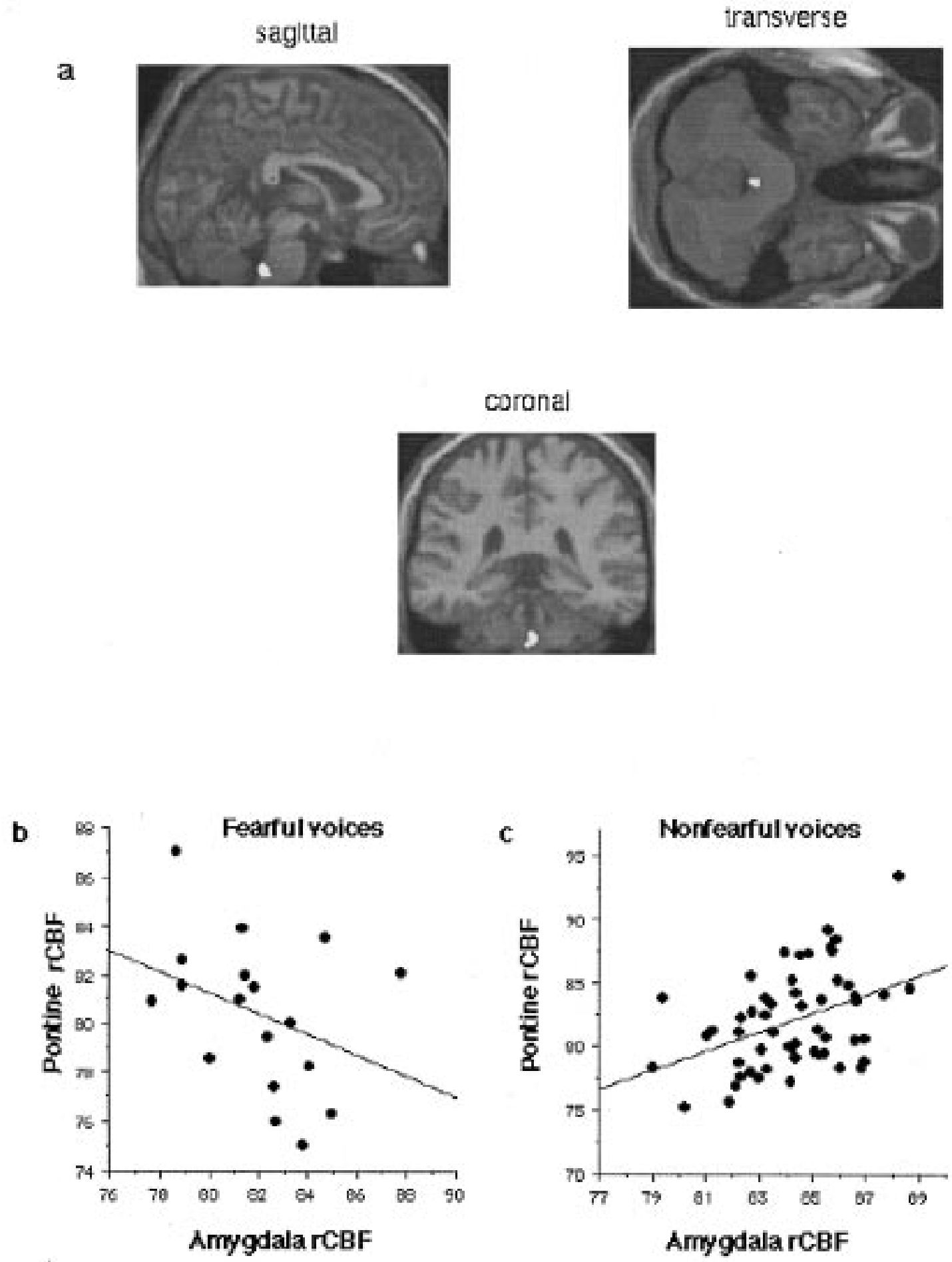

Fig. 3. (a) An SPM showing a region of the pons where there is a significant $(P<0.01)$ fear-specific change of covariation with right amygdala activity. The activation is displayed on orthogonal slices of a canonical MRI centred on the maximally activated voxel $(x=2, y=-36, z=-38)$. An uncorrected $P$ value of 0.01 was used as the threshold for the contrast; (b) bivariate regression plot of $r \mathrm{CBF}$ in the maximally activated voxel in the pons ( $x=2, y=-36, z=-38)$ during presentation of fearful voices; (c) bivariate regression plot of $r$ CBF in the maximally activated voxel in the pons $(x=2, y=-36, z=-38)$ during presentation of happy, sad and neutral voices.

ging [12] data have tended to show right hemisphere dominance for emotional prosody, some of our results show left lateralization of responses. Lesion evidence of left hemisphere involvement in emotional prosody
$[6,7,15,17]$ has been interpreted in terms of inter-hemispheric interactions required to integrate the rightsided prosodic and left-sided propositional content of speech. However, since we used entirely non-verbal 
stimuli, our results cannot be attributed simply to left hemisphere language specialization. Left-sided dominance for vocal stimuli in general, and a left hemisphere (particularly left insula) specialization [14,27,29] for the temporal ordering of auditory information may provide other explanations for the lateralization. Leftsided lesions appear to have a greater effect on the recognition of negative vocal emotion [27]. This finding is consistent with the pattern of left-sided insula responses to fearful and sad voices observed in the present study (Fig. 1 (c) and (d)).

It is possible that the explicit sex decision task performed by subjects during scanning may have biased responses to the left hemisphere. Behavioural experiments have shown that classification of faces into male and female involves a strong left hemisphere dominance (particularly in males), even though face discrimination in general is more associated with the right hemisphere $[18,19]$. A neuroimaging study of healthy male subjects performing a sex decision task with fearful and happy faces found responses strongly lateralized to the left amygdala [25]. The vocal sex decision task in the present study may also have preferentially engaged the left hemisphere of the exclusively male subjects, thus producing the lateralized results. It is possible that a different pattern of neural activity would be seen in female subjects.

The fear-related amygdala activity in the present study (Table $2 b$ and Fig. $2 b$ ) is consistent with lesion data showing a selective recognition deficit for fearful vocalizations in a patient with bilateral amygdala damage [37]. Furthermore, an association between right amygdala activity and fearful vocalizations has been previously reported by another neuroimaging study, although the response in the amygdala was described as an 'activation' rather than a decrease in activity [28]. However, the method of data analysis used in the other experiment [28] did not distinguish the sign of the response, and treated all condition-related deviations from baseline activity, either negative or positive, as 'activations'. It is possible, therefore, that the fear-specific decrease in right amygdala response observed in the present experiment is equivalent to the other study's 'activation'.

The decreased amygdala response to fearful voices does, however, contrast with the previously observed increased amygdala responses to fearful facial expressions $[4,25]$. There are several possible explanations for these differing results. It is known from electrophysiological studies $[30,32,33]$ that amygdala cells have complex response patterns to afferent stimulation. Typical responses (c. 75\% of all cells) involve multiphasic excitatory and inhibitory activity; pure excitatory or inhibitory responses are less common. Electrophysiological [31] and neuroimaging studies $[5,20]$ have also revealed that amygdala responses ha- bituate rapidly. Since PET integrates neural activity across $90 \mathrm{~s}$, mean recorded amygdala activity depends crucially on temporal response dynamics, e.g. non-linear response summation as a function of stimulus frequency, the effect of blocked vs random stimulus presentation and habituation across session. Differential auditory and visual response dynamics in the amygdala for fearful stimuli may explain, therefore, the contrasting changes in mean activity between the two sensory modalities.

The decrease in amygdala activity observed in the fearful condition may also have resulted from extrinsic inhibitory modulation. Behavioural and electrophysiological data provide evidence that the amygdala is subject to inhibitory modulation by frontal regions [22,39]. In keeping with these findings, we observed that the anterior insula, which selectively increased its activity during fearful voice presentation, exhibited a fear-specific inhibitory interaction with the right amygdala. Although these data are suggestive of functional interactions between amygdala and insula, further investigation is required to determine the nature of the mechanisms involved.

Several subjects, during post-scan debriefing, spontaneously reported that the fearful voices were 'startling'. One of the best studied phenomena in fear processing, the acoustic startle reflex, is mediated by a pathway in the brainstem involving the ventral cochlear nucleus, ventral nucleus of lateral lemniscus and the nucleus reticularis pontis caudalis (NRPC) [8]. Fear-related modulation of the startle reflex has been shown in animal studies to involve a direct projection from amygdala to NRPC [8,38]. The fear-specific interaction between the right amygdala and a region of the pons with a maximal focus coincident with the NRPC accords, therefore, with the known anatomy of descending amygdala projections. Our data imply that brain regions involved in acoustic startle interact with systems mediating auditory affective processing, providing evidence, therefore, of functional integration across different neural networks.

In conclusion, we have identified a distributed neural system responsive to emotional vocalizations involving pontine, caudate and amygdala nuclei, as well as insula, temporal and ventral prefrontal cortices. Within this system, specific differential responses to fearful vocal sounds were seen in right amygdala and left insula. Regression analyses based on the neural activity in these regions identified the presence of fearspecific interactions between the anterior insula, amygdala, and a pontine region (NRPC) implicated in acoustic startle. The findings are broadly consistent with human lesion data on aprosodia and animal experiments on fear-potentiated startle, but they fail to support models of vocal emotion processing in which only the right hemisphere has a significant role. 
Rather, the data suggest that processing of emotional vocalizations requires not only functional specialization, e.g. selective amygdala and insula responses to fearful voices, but also the integration of neural activity across left and right hemispheres and the brainstem.

\section{Acknowledgements}

J.S.M. and R.J.D. are supported by the Wellcome Trust. We thank Andrew Calder and Andrew Young for their assistance with the study.

\section{References}

[1] Adolphs R, Tranel D, Damasio H, Damasio AR. Fear and the human amygdala. Journal of Neuroscience 1995;15:5879-91.

[2] Amaral DG, Price JL, Pitkanen A, Carmichael ST. Anatomical organization of the primate amygdaloid complex. In: Aggleton JP, editor. The amygdala: neurobiological aspects of emotion, memory and mental dysfunction. New York: Wiley-Liss, 1992. p. 1-66.

[3] Augustine JR. Circuitry and functional aspects of the insular lobe in primates including humans. Brain Research Reviews 1996;22:229-44.

[4] Breiter HC, Etcoff NL, Whalen PJ, Kennedy DN, Rauch SL, Buckner RL, Strauss MM, Hyman SE, Rosen BR. Response and habituation of the human amygdala during visual processing of facial expression. Neuron 1996;2:875-87.

[5] Büchel C, Morris JS, Dolan RJ, Friston KJ. Brain systems mediating aversive conditioning: an event-related fMRI study. Neuron 1998;20:947-57.

[6] Cancelliere AEB, Kertesz A. Lesion localization in acquired deficits of emotional expression and comprehension. Brain and Cognition 1990;13:133-47.

[7] Darby DG. Sensory aprosodia: a clinical clue to lesions of the inferior division of the right middle cerebral artery? Neurology 1993;43:567-72.

[8] Davis M. The amygdala and conditioned fear. In: Aggleton JP, editor. The amygdala: neurobiological aspects of emotion, memory and mental dysfunction. New York: Wiley-Liss, 1992. p. 255-306.

[9] Dronkers NF. A new brain region for coordinating speech articulation. Nature 1996;384:159-61.

[10] Friston KJ, Büchel C, Fink G, Morris JS, Rolls ET, Dolan RJ. Psychophysiological and modulatory interactions in neuroimaging. NeuroImage 1997;6:218-29.

[11] Friston KJ, Holmes AP, Worsley KJ, Poline J-P, Frith CD, Frackowiak RSJ. Statistical parametric maps in functional imaging: a general linear approach. Human Brain Mapping 1995;2:189-210.

[12] George MS, Parekh PI, Rosinsky N, Ketter TA, Kimbrell, Heilman KM, Herscovitch P, Post RM. Understanding emotional prosody activates right hemisphere regions. Archives of Neurology 1996;53:665-70.

[13] Gorelick PB, Ross ED. The aprosodias: further functional-anatomical evidence for the organization of affective language in the right hemisphere. Journal of Neurology Neurosurgery \& Psychiatry 1987;50:553-60.

[14] Guldin WO, Markowitsch HJ, Lampe R, Irle E. Cortical projections originating from the cat's insular area and remarks on claustrocortical connections. Journal of Comparative Neurology 1986;243:468-87.

[15] Habib M, Daquin G, Milandre L, Royere ML, Rey M, Lanteri A, Salamon G, Khalil R. Mutism and auditory agnosia due to bilateral insular damage - role of the insula in human communication. Neuropsychologia 1995;33:327-39.

[16] Heilman KM, Scholes R, Watson RT. Auditory affective agnosia. Disturbed comprehension of affective speech. Journal of Neurology Neurosurgery \& Psychiatry 1975;38:69-72.

[17] Hornak J, Rolls ET, Wade D. Face and voice expression identification in patients with emotional and behavioural changes following ventral frontal lobe damage. Neuropsychologia 1996;34:247-61.

[18] Jones B. Sex and visual field effects on accuracy and decision making when subjects classify male and female faces. Cortex 1979;15:551-60.

[19] Jones B, Anuza T. Sex differences in cerebral lateralization in 3 and 4 year old children. Neuropsychologia 1982;20:347-50.

[20] LaBar KS, Gatenby JC, Gore JC, LeDoux JE, Phelps EA. Human amygdala activation during fear acquisition and extinction: a mixed trial fMRI study. Neuron 20:937-945.

[21] LeDoux JE. In search of an emotional system in the brain: leaping from fear to emotion and consciousness. In: Gazzaniga MS, editor. The cognitive neurosciences. Cambridge, MA: The MIT Press, 1995. p. 1049-61.

[22] Al-Maskati HA, Zbrozyna AW. Stimulation in prefrontal cortex area inhibits cardiovascular and motor components of the defence reaction in rats. Journal of the Autonomic Nervous System 1989;28:117-25.

[23] Monrad-Krohn GH. Dysprosody or altered 'melody of language'. Brain 1947;70:405-15.

[24] Morris JS, Friston KJ, Dolan RJ. Neural responses to salient visual stimuli. Proceedings of the Royal Society of London Series B 1997;264:760-75.

[25] Morris JS, Frith CD, Perrett DI, Rowland D, Young AW, Calder AJ, Dolan RJ. A differential neural response in the human amygdala to fearful and happy facial expressions. Nature 1996;383:812-5.

[26] Murray IR, Arnott JL. Towards the simulation of emotion in synthetic speech: a review of the literature on human vocal emotion. Journal of the Acoustical Society of America 1993;93:1097-108.

[27] Pell MD. Recognition of prosody following unilateral brain lesion: influence of functional and structural attributes of prosdic contours. Neuropsychologia 1998;36:701-15.

[28] Philips ML, Young AW, Scott SK, Calder AJ, Andrew C, Giampietro V, Williams SCR, Bullmore ET, Brammer M, Gray JA. Neural responses to facial and vocal expressions of fear and disgust. Proceedings of the Royal Society of London Series B 1997;265:1809-17.

[29] Platel H, Price C, Baron J-C, Wise R, Lambert J, Frackowiak RJS, Lechevalier B, Eustache F. The structural components of music perception. A functional anatomical study. Brain 1997; 120:229-43.

[30] Prelevic S, McIntyre Burnham W, Gloor P. A microelectrode study of amygdaloid afferents: temporal neocortical units. Brain Research 1976;105:437-57.

[31] Quirk GJ, Armony JL, LeDoux JE. Fear conditioning enhances different temporal components of tone-evoked spike trains in auditory cortex and lateral amygdala. Neuron 1997;19:613-24.

[32] Rainnie DG, Asprodini EK, Shinnick-Gallagher P. Excitatory transmission in the basolateral amygdala. Journal of Neurophysiology 1991;66:986-98.

[33] Rainnie DG, Asprodini EK, Shinnick-Gallagher P. Inhibitory transmission in the basolateral amygdala. Journal of Neurophysiology 1991;66:999-1009. 
[34] Ross ED, Anderson B, Morgan-Fisher A. Crossed aprosodia in strongly dextral patients. Archives of Neurology 1989;46:206-9.

[35] Ross ED, Harney JH, deLacoste-Utamsing C, Purdy PD. How the brain integrates affective and propositional language into a unified behavioural function. Hypothesis based on clinicoanatomic evidence. Archives of Neurology 1981;38:745-8.

[36] Ryalls J. Concerning right-hemisphere dominance for affective language. Archives of Neurology 1988;45:337-8.

[37] Scott SK, Young AW, Calder AJ, Hellawell DJ, Aggleton JP,
Johnson M. Impaired auditory recognition of fear and anger following bilateral amygdala lesions. Nature 1997;385:254-7.

[38] Wan F-J, Swerdlow NR. The basolateral amygdala regulates sensorimotor gating of the acoustic startle in the rat. Neuroscience 1997;76:715-24.

[39] Zbrozyna AW, Westwood DM. Stimulation in prefrontal cortex inhibits conditioned increase in blood pressure and avoidance bar pressing in rats. Physiology \& Behaviour 1991;49:705-8. 